\title{
Load and Deflection Measurement for Evaluation of Ground Strength with Portable FWD System
}

\author{
Takahiro Fujyu*, Jun Sugawara*, Hiroyuki Takuno* and Haruki OKano*
}

\begin{abstract}
In portable falling weight deflectometer (FWD-Light) tests, the impact load and the deflection generated by means of a weight dropping on a loading plate are measured by the strain gauge type load cell and accelerometer, and are applied to nondestructive evaluation of the ground strength. In this paper, the techniques used in the load and the deflection measurements in the FWD-Light system are presented.
\end{abstract}

Key Words: FWD-Light, load, deflection, ground strength

\section{Introduction}

A portable falling weight deflectometer (FWD-Light) is a nondestructive testing system used for evaluation of the ground strength. It consists of ( 1 ) a main body, (2) an external deflection module, and (3) an indicator with a built-in data processing system (Fig. 1). Furthermore, the main body can be subdivided as follows:

- a weight for generating the impact load.

- a guiding rod for freely falling of the weight onto rubber buffers.

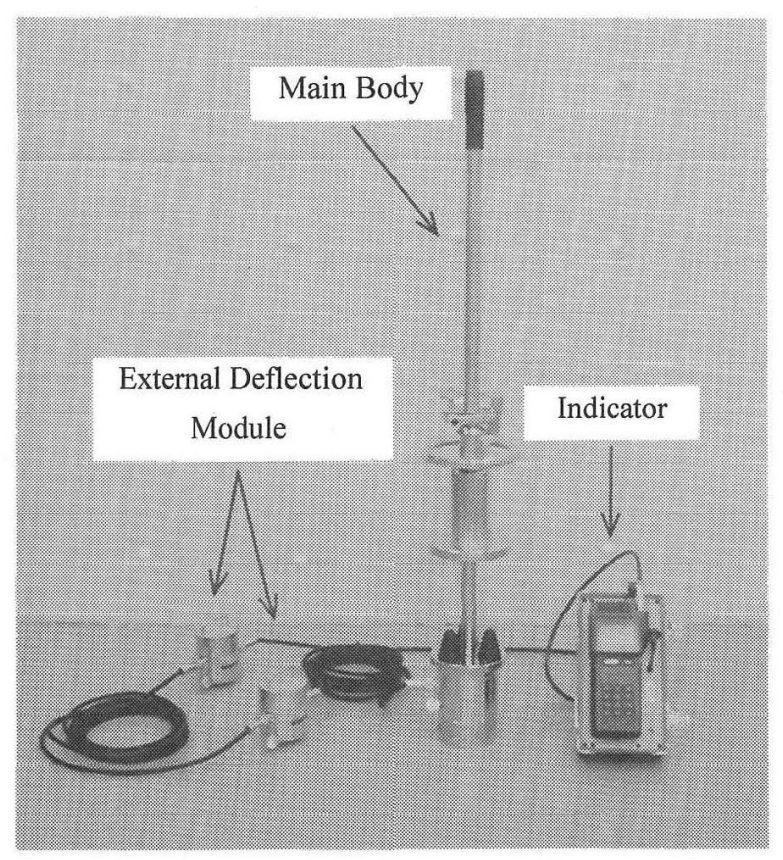

Fig. 1 FWD-Light system

\footnotetext{
* Tokyo Sokki Kenkyujo Co., Ltd., Aioi, Kiryu

(Received March 8, 2004)

(Revised June 18, 2004)
}

- a lever with a stopper for holding and releasing the weight.

- rubber buffers for absorbing the impact load.

- a load cell for measuring the impact load.

- an accelerometer for measuring the deflection.

- a casing with built-in modules for transferring sensor outputs to the indicator through a 2-wire digital line, and

- a loading plate set under the casing of the main body for transferring the impact load to the ground.

The FWD-Light system features excellent portability due to small size and light weight. Table 1 shows specifications of the system.

In this system, the impact load generated by means of a weight dropping on the loading plate set on the ground

Table 1 Specifications of FWD-Light system

\begin{tabular}{c|c}
\hline \hline $\begin{array}{c}\text { Dimensions of loading } \\
\text { plate }\end{array}$ & $0.1,0.2$, and $0.3 \mathrm{~m}$ \\
\hline Mass of weight & 5,10, and $15 \mathrm{~kg}$ \\
\hline Falling height (variable) & $0.05 \sim 0.55 \mathrm{~m}$ \\
\hline Maximum load & $\sim 20 \mathrm{kN}$ \\
\hline Maximum deflection & $2.5 \times 10^{-3} \mathrm{~m}$ \\
\hline Sensors & Load cell and accelerometer \\
\hline No. of measuring points & $\begin{array}{c}2 \text { points } \\
\text { (load and acceleration) }\end{array}$ \\
\hline Data memory & 800 data/point \\
\hline Sampling speed & By load data \\
\hline Trigger function & Exclusive 2 -wire serial transfer \\
\hline Interface & Maximum 2 points \\
\hline $\begin{array}{c}\text { No. of external } \\
\text { deflection modules }\end{array}$ & Approx. $1.1 \mathrm{~m}$ \\
\hline Height & Approx. $15 \mathrm{~kg}$ \\
\hline Weight & (including $5 \mathrm{~kg}$ mass of weight) \\
\hline
\end{tabular}


surface is measured by the strain gauge type load cell. The deflection of the ground is determined by double integration of acceleration. As well, the deflection under the external deflection module is obtained in the same manner. No reference fixed point is required in the deflection measurement since strain gauge type accelerometers are employed in the system.

Analogue outputs of each sensor are converted into digital data by an $\mathrm{A} / \mathrm{D}$ converter which is placed near the sensor and are transferred to the indicator. Moreover, a cylindrical metallic casing of the main body functions as a noise shield since it accommodates the load cell, the accelerometer, and the $\mathrm{A} / \mathrm{D}$ converter inside. As a result, this technique effectively prevents data from noise being intruded during the data transfer and yields the reliable measurement results.

\section{Measurement of Load and Acceleration}

\subsection{Data scanning and collection}

Fig. 2 shows an outline of modules for the load cell and the accelerometer in the FWD-Light system. When the data processing system gives instructions of a measurement start to the modules under the condition that the module circuit is on, voltage power is supplied from the bridge power circuit to the Wheatstone bridge circuit of the load cell module. Digital outputs of the load cell and the accelerometer are then acquired through the amplifier and the $\mathrm{A} / \mathrm{D}$ converter at the sampling speed of $50 \mu \mathrm{s}$. Data corresponding to the predetermined period (e.g., 20 ms) are updated and stored in the pre-memory area of the memory circuit in series on time.

At every sampling instant, CPU of the load cell module computes the difference between the current digital output of the load cell $\mathrm{F}(k)$ and the last value $\mathrm{F}(k-1)$. Then CPU compares the difference $(\mathrm{F}(k)-\mathrm{F}(k-1))$ with the predetermined value $\Delta \mathrm{f}$ which is slightly bigger than zero. CPU repeats this process until the value of $(\mathrm{F}(k)-\mathrm{F}(k$ $-1)$ ) becomes larger than $\Delta$ f, i.e., until the weight of the

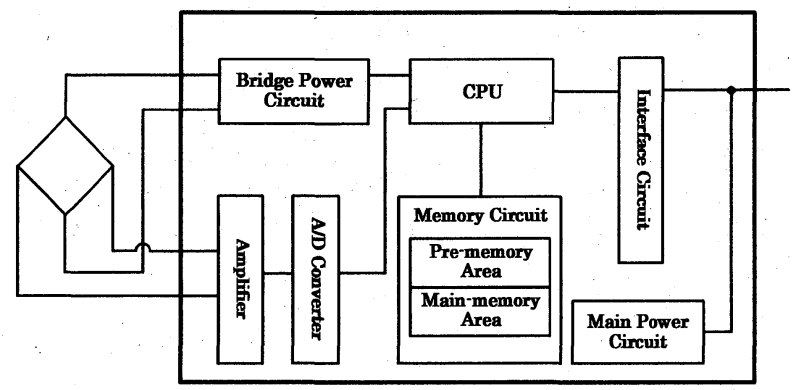

Fig. 2 Outline of load cell and accelerometer modules
FWD-Light system impacts rubber buffers.

When the weight impacts rubber buffers, the ground under the loading plate deflects downwards and as a result acceleration is generated. In this case, outputs of the load cell and the accelerometer vary as shown in Fig. 3 (a) and $3(\mathrm{~b})$, respectively. When the value of $(\mathrm{F}(k)-\mathrm{F}(k-1))$. exceeds $\Delta \mathrm{f}$ at the time $t_{n}$ shown in Fig. 3 (a) and 4, CPU of the load cell module starts accumulating the value of $(\mathrm{F}(k)-\mathrm{F}(k-1))$ for every sampling instant.

The accumulated value $\left(\sum(\mathrm{F}(k)-\mathrm{F}(k-1))\right)$ is compared with the predetermined trigger level TL: TL is a value

(a)
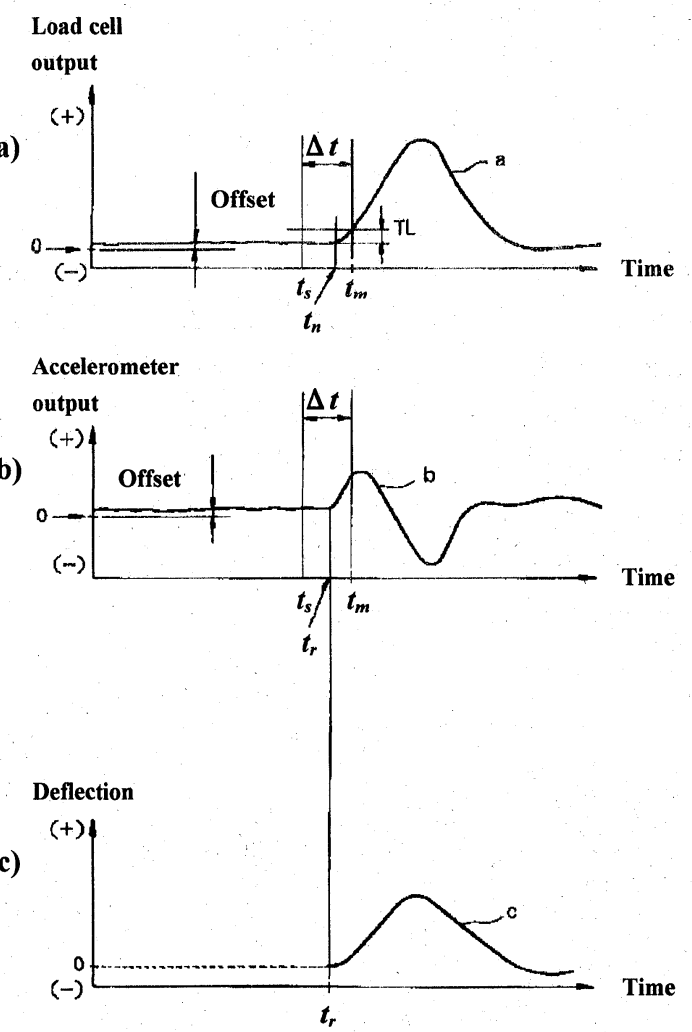

Fig. 3 Relationships between ( a ) load cell output and time, (b) accelerometer output and time, and (c) deflection and time

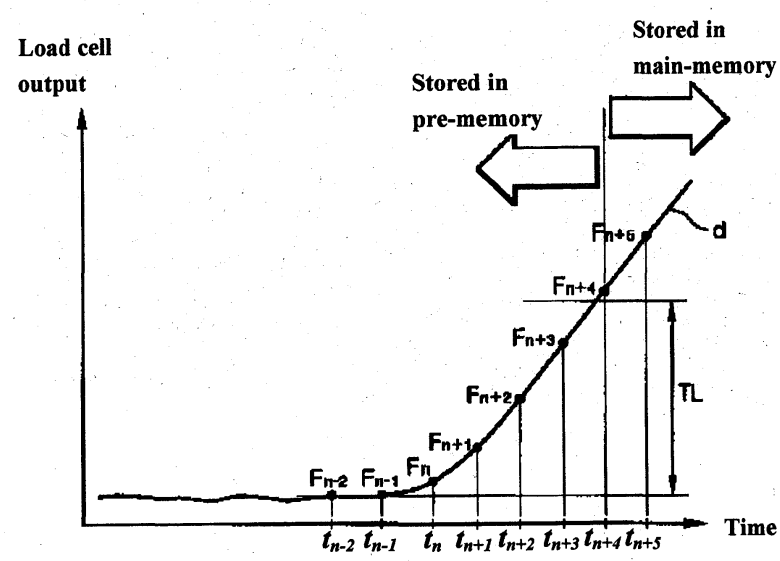

Fig. 4 Relationship between load cell output and time 
bigger than $\Delta \mathrm{f}$ and is typically $1 \mathrm{kN}$ in the system. When the accumulated value $(\Sigma(\mathrm{F}(k)-\mathrm{F}(k-1)))$ becomes larger than TL, CPU of the load cell module gives a trigger signal to CPU of the acceleration module. Then digital data in the pre-memory area of the memory circuit are retained as a part of measurement data. This point in time is called the trigger timing and is denoted as $t_{m}$ in Fig. $3(\mathrm{a})$ and $3(\mathrm{~b})$. In addition, digital data corresponding to the predetermined period (e.g., $20 \mathrm{~ms}$ ) after $t_{m}$ are stored at the main-memory area of the memory circuit in series on time. In this way, digital data from the load cell and the accelerometer are stored in the memory circuit as load measurement data and acceleration measurement data, respectively.

\section{2 Data offset}

Due to circuit characteristics or noises, output signals of the load cell and the accelerometer may shift from the zero-level even before the weight impacts on rubber buffers. CPU in modules therefore needs to carry out the process called the offset removal processing in order to eliminate the signal offset from measurement data.

In this process, CPU of the load cell module determines an average value of load measurement data as the offset level. Load measurement data obtained before the time $t_{s}$ in Fig. 3( $\mathrm{a}$ ) is used in this calculation. It is noted that $t_{s}$ is the time before the weight impacts on rubber buffers, i.e., before the output of the load cell starts increasing. CPU of the load cell module subtracts the offset level from measurement data in the pre-memory and the main-memory of the memory circuit. Then data obtained in this process are restored as load measurement data. In the same manner, the offset removal processing of acceleration measurement data is carried out at the acceleration module.

\section{Determination of Deflection}

The deflection of the ground is determined by double integration of acceleration measurement data. Data measured after the standing up timing which is defined as the time that acceleration measurement data starts increasing from the zero-level are used in this process.

\subsection{Standing up timing determination}

In accordance with the steps shown in Fig. 5, the standing up timing $t_{r}$ of acceleration measurement data is determined. In this flow chart, $p$ is an integer and $\mathrm{a}(p)$ represents acceleration measurement data obtained at the sampling time $t_{p}$. It is noted that $\mathrm{a}(p)$ goes back to a past side as the value of $p$ decreases.

In the determination process of the standing up timing,

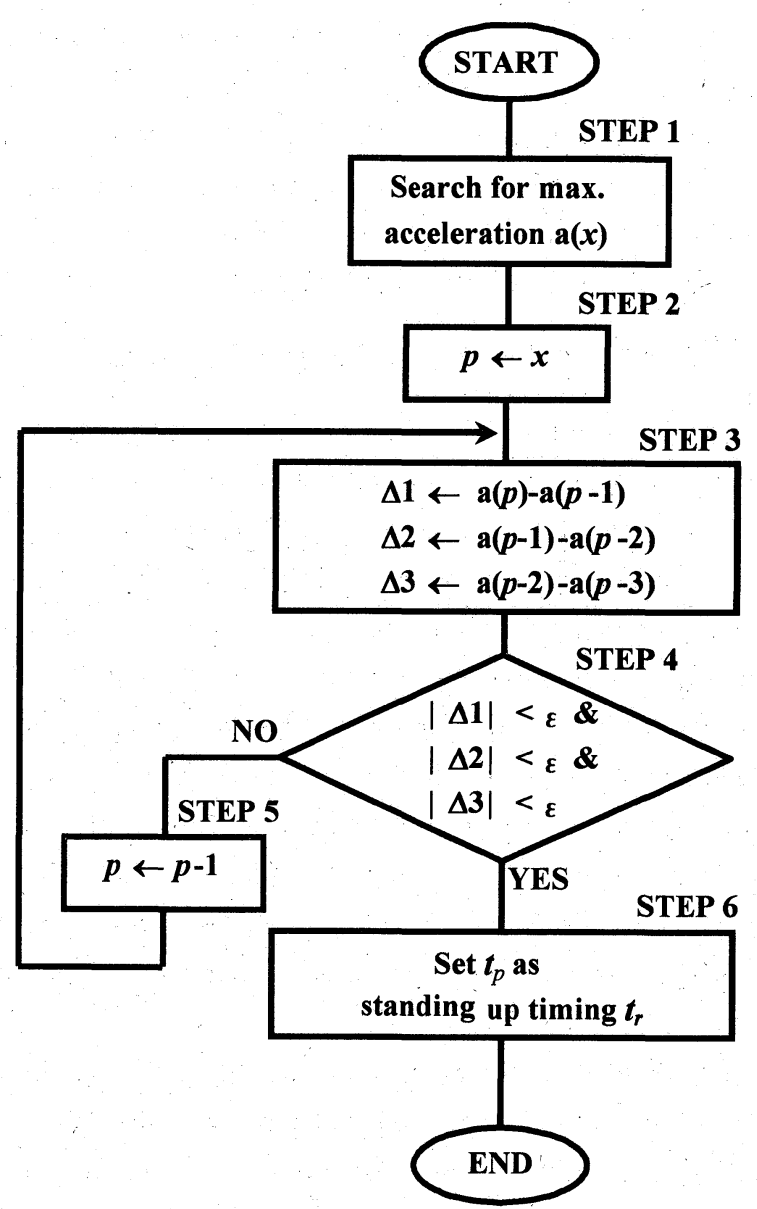

Fig. 5 Flow chart for determination of standing up timing

$\mathrm{CPU}$ of the acceleration module searches for the maximum value $\mathrm{a}(x)$ in the acceleration measurement data stored at the memory circuit (STEP 1). The integer $p$ is initialized to the integer $x$. It should be noted that integer $x$ is the value used in the expression of the maximum acceleration measured data $\mathrm{a}(x)$ at the time $t_{x}$ (STEP 2). CPU extracts consecutive acceleration measurement data, e.g., $\mathrm{a}(p), \mathrm{a}(p-1), \mathrm{a}(p-2)$, and $\mathrm{a}(p-3)$ and determines the differences $\Delta 1=\mathrm{a}(p)-\mathrm{a}(p-1), \Delta 2=\mathrm{a}(p-1)-\mathrm{a}(p-2), \Delta 3=$ $\mathrm{a}(p-2)-\mathrm{a}(p-3)$ (STEP 3$)$. CPU examines if absolute values of $\Delta 1, \Delta 2$, and $\Delta 3$ are smaller than the predetermined value $\varepsilon$ which is a positive value being approximately zero (STEP 4). If the conditions. $|\Delta 1|<\varepsilon,|\Delta 2|<\varepsilon$, and $|\Delta 3|<\varepsilon$ are not satisfied, the value of $p$ is replaced by the value $p-1$ (STEP 5 ). Then steps from STEP 2 are repeated. When $|\Delta 1|<\varepsilon,|\Delta 2|<\varepsilon$, and $|\Delta 3|<\varepsilon$ are satisfied in STEP 4, CPU sets the time $t_{p}$ as the standing up timing $t_{r}$ (STEP 6).

\subsection{Integration}

Based on a trapezoid formula, CPU in the accelerometer module performs double integration of acceleration measurement data obtained after the standing up timing 
$t_{r}$. Then results are restored at the pre-memory and the main-memory of the memory circuit of the acceleration module as the deflection of the ground in series on time. An example of the ground deflection determined in this process is given in Fig. 3(c). The downward ground deflection is usually taken as positive.

\section{Evaluation of Ground Strength}

CPU in modules transmits load and deflection measurement data from the menory circuit to the data processing system in series on time. Firstly, CPU sends out either load or deflection measurement data through an interface circuit and a cable which consists of a common signal line. Then CPU sends out another data to the data processing system. It should be pointed out that the power to each module is also supplied through the abovementioned signal line since data between the data processing system and modules are in a digital form. Therefore, the cable in the FWD-Light system can be composed of only a few signal lines, contributing to cable flexibility as well as improved workability.

$K_{T M L}$ defined as the coefficient of subgrade reaction by FWD-Light tests is determined according to the approach used in the plate loading test. $K_{\text {TML }}$ is obtained by the stress generated due to the impact of a falling weight divided by the deflection corresponding to the loading plate whose diameter is $0.3 \mathrm{~m}$ :

$$
K_{T M L}=\left(\frac{P_{\phi}}{\delta_{\phi}}\right) \cdot\left(\frac{\phi}{\phi_{P L T}}\right)
$$

where, $\phi$ is the diameter of the loading plate, $P_{\phi}$ is the maximum stress measured with the loading plate whose diameter is $\phi, \delta_{\phi}$ is the maximum deflection measured with the loading plate whose diameter is $\phi$, and $\phi_{P L T}$ is the diameter of the loading plate used in the plate loading test $(0.3 \mathrm{~m})$.

Moreover, $E_{T M L}$ defined as Young's modulus by FWDLight tests is obtained based on Burmister's theoretical solution :

$$
E_{T M L}=\frac{2 \cdot\left(1-\nu^{2}\right) \cdot F_{\phi}}{\pi \cdot r \cdot \delta_{\phi}}
$$

where, $\nu$ is Poisson's ratio, $F_{\phi}$ is the maximum load measured with the loading plate whose diameter is $\phi$, and $r$ is the radius of the loading plate. It is noted that typical values of Poisson's ratio are : sandy soils $=0.3 \sim 0.4$, cohesive soils $=0.25 \sim 0.45$, and volcanic cohesive soils $=0.4 \sim$ 0.5 .

Evaluation results are displayed at the indicator of the FWD-Light system with the maximum load, the maximum deflections under the loading plate and the external

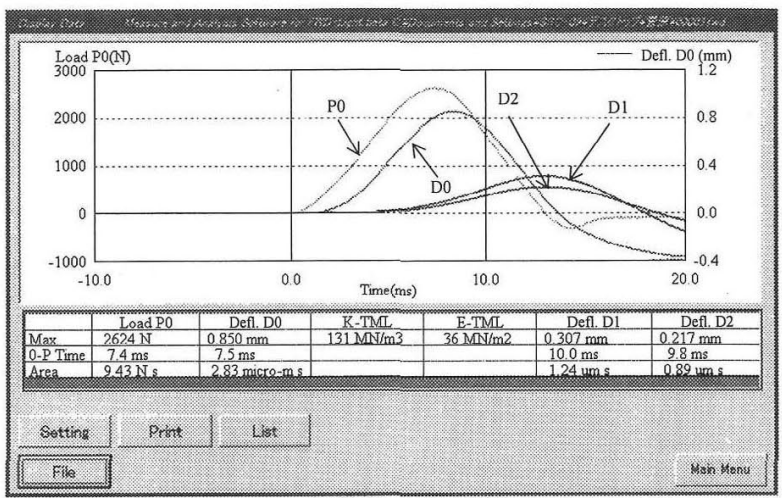

Fig. 6 Example of waveform indication using measurement/ analyses software

deflection module. Moreover, in addition to the display by the indicator, the load and deflections are displayed in the waveform by using the exclusive measurement/analyses software. Furthermore, $0-\mathrm{P}$ time defined as the time from the standing up timing to the maximum value of the load or the deflection measurement data, and the time product of waveforms defined as the area under load or deflection curve is also displayed on the same display (Fig. 6).

\section{Conclusions}

This paper presented the techniques used in the load and the deflection measurements in the FWD-Light system.

In the system, outputs of each sensor are digitized by the internal $\mathrm{A} / \mathrm{D}$ converter, and those measured near the trigger timing are stored at the memory. Then outputs of the accelerometer recorded after the standing up timing are integrated twice for determination of the deflection. Then results of measurements and analyses are used for nondestructive evaluation of the ground strength.

We believe that investigating relationships between the FWD-Light test and other in-situ tests including the plate loading test and the field CBR test promise to be a fruitful area of future research.

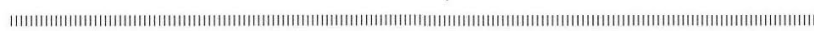
Takahiro Fujyu

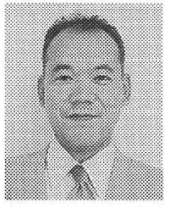

Takahiro Fujyu received B.S. degree in mathematics from Tokyo University of Sci ence in Japan in 1985. He joined Tokyo Sokki Kenkyujo Co., Ltd. in 1989. Since then, he has been engaged in research and development of strain gauge type sensors and portable FWD system. 
Jun Sugawara

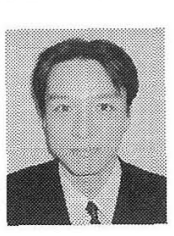

Jun Sugawara received B.E. degree in mechanical engineering from Meiji University in Japan in 1991, M.E. degree in civil and environmental engineering from Carleton University in Canada in 1999, and Ph.D. degree in civil engineering from the University of Hong Kong in 2002. Currently he works at Tokyo Sokki Kenkyujo Co., Ltd. as a research engineer. His research interests include geo-instrumentation and ground characterization using potable FWD system.

\section{Hiroyuki TAKUno}

Hiroyuki Takuno received B.E. and M.E.

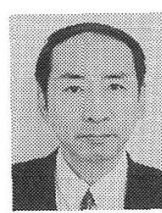

degrees in civil engineering from Kyushu Insti tute of Technology in Japan in 1976 and 1978, respectively. He joined Tokyo Sokki Kenkyu jo Co., Ltd. in 1978. Since then, he has been engaged in research and development of strainmeters.

\section{Haruki OKano (Member)}

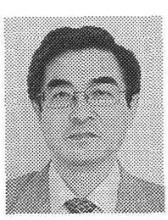

Haruki Okano graduated from Shimodate Technical High School in Japan in 1968. He joined Tokyo Sokki Kenkyujo Co., Ltd. in 1969 and has been mainly engaged in research and development of strainmeters. He received awards from The Japan Society for the Advancement of Inventions, and The Nikkan Kogyo Shimbun, Ltd. in 2001, and Ministry of Education, Culture, Sports, Science and Tech nology in 2002 for research on Complete Com pensation Method of Strain (CCMS). Since 2002, he has been a factory manager and a director of the company.

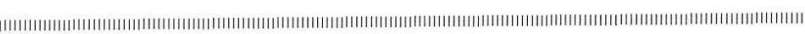

